Supporting Information

\title{
Borophene-Based Three-Dimensional Porous Structures as Anode Materials for Alkali Metal-Ion Batteries with Ultrahigh Capacity
}

Imran Muhammad ${ }^{\mathrm{a}}$, Umer Younis ${ }^{\mathrm{a}}$, Huanhuan Xie ${ }^{\mathrm{a}}$, Adnan Ali Khan ${ }^{\mathrm{b}}$, Abdul Khaliq ${ }^{\mathrm{c}}$, Abdus Samad ${ }^{\mathrm{d}}$, Udo Schwingenschlögl ${ }^{\mathrm{d}}$, and Qiang Sun ${ }^{\mathrm{a}, *}$

${ }^{a}$ School of Materials Science and Engineering, CAPT, Peking University, Beijing, China

${ }^{b}$ Department of Chemistry, University of Malakand, Chakdara, Lower Dir, 18800, Pakistan.

${ }^{c}$ Institute of Physics, Polish Academy of Sciences, Aleja Lotnikow 32/46 Warsaw (02-668) Poland.

${ }^{d}$ Physical Science and Engineering Division (PSE), King Abdullah University of Science and Technology (KAUST), Thuwal 23955-6900, Saudi Arabia.
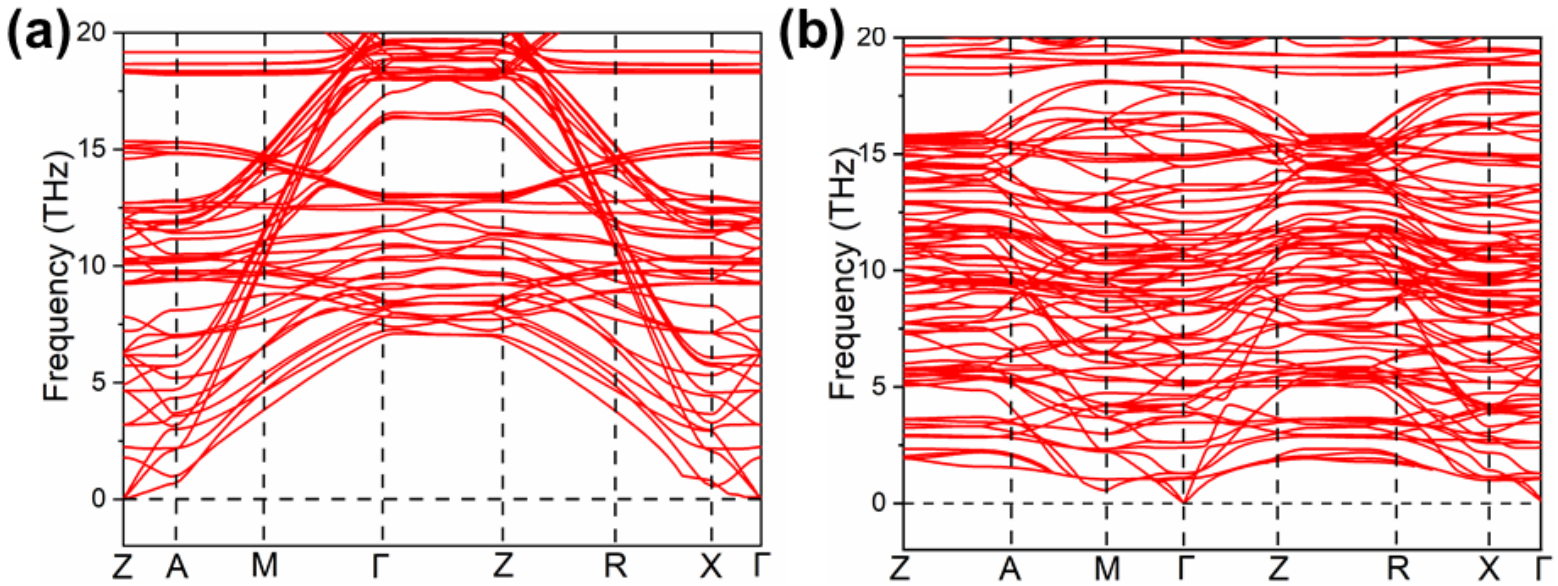

Figure S1. Phonon band structures of (a) $3 \mathrm{D}-\beta_{12}$-borophene and (b) $3 \mathrm{D}-\mathrm{B}_{7} \mathrm{P}_{2}$.

Table S1. Independent Elastic Constants $\left(C_{i j}\right)$, Bulk Moduli $(B)$, Shear Moduli $(G)$, Young's moduli $(E)$, and Pugh Ratios $(\mathrm{B} / \mathrm{G})$ of $3 \mathrm{D}-\beta_{12}$-borophene and $3 \mathrm{D}-\mathrm{B}_{7} \mathrm{P}_{2}$.

\begin{tabular}{|c|c|c|c|c|c|c|c|c|c|c|c|c|c|}
\hline & $C_{11}$ & $C_{22}$ & $C_{33}$ & $C_{44}$ & $C_{55}$ & $C_{66}$ & $C_{12}$ & $C_{13}$ & $C_{23}$ & $B$ & $G$ & $E$ & $B / G$ \\
\hline $\begin{array}{c}\text { 3D- } \beta_{12-} \\
\text { borophene }\end{array}$ & 135.7 & 137.0 & 396.4 & 57.6 & 60.3 & 59.2 & 62.5 & 21.5 & 21.8 & 101.9 & 65.8 & 162.3 & 1.5 \\
\hline $3 D-B_{7} P_{2}$ & 69.7 & 180.8 & 70.1 & 39.3 & 43.7 & 8.51 & 18.0 & 43.5 & 18.1 & 105.5 & 31.3 & 85.4 & 3.4 \\
\hline
\end{tabular}



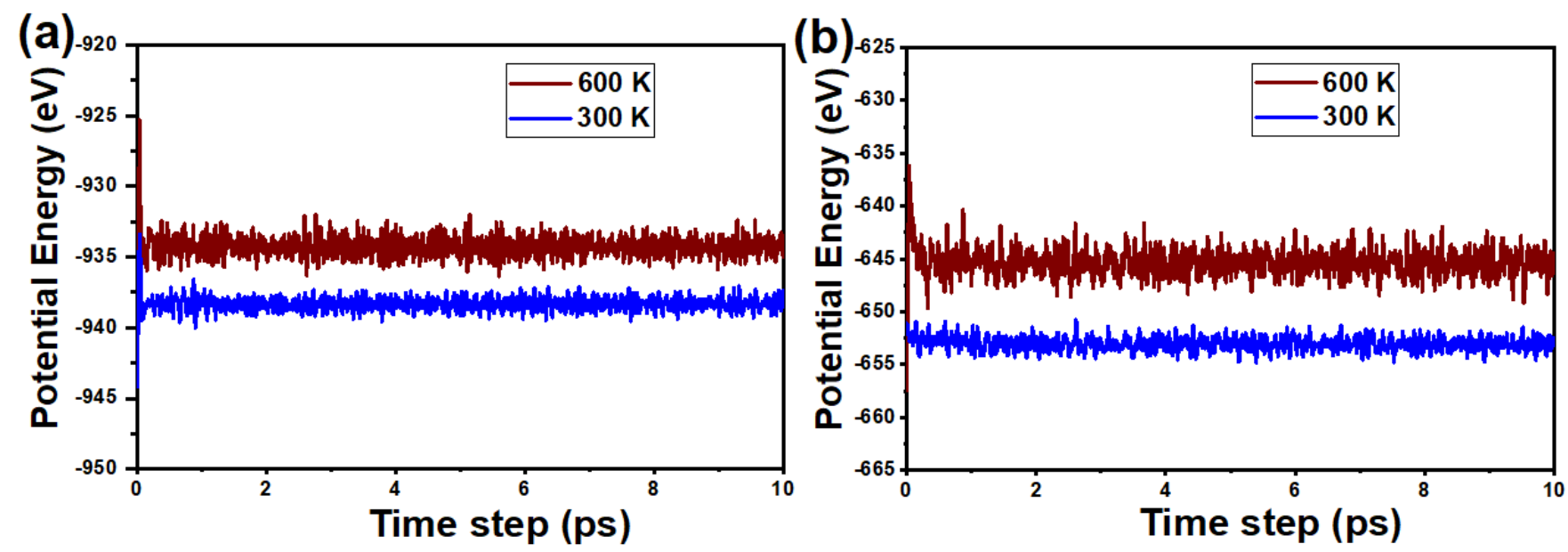

Figure S2. Fluctuations of the potential energy for (a) $3 \mathrm{D}-\beta_{12}$-borophene and (b) $3 \mathrm{D}-\mathrm{B}_{7} \mathrm{P}_{2}$ during $10 \mathrm{ps}$ AIMD simulations at temperatures of 300 and $600 \mathrm{~K}$.

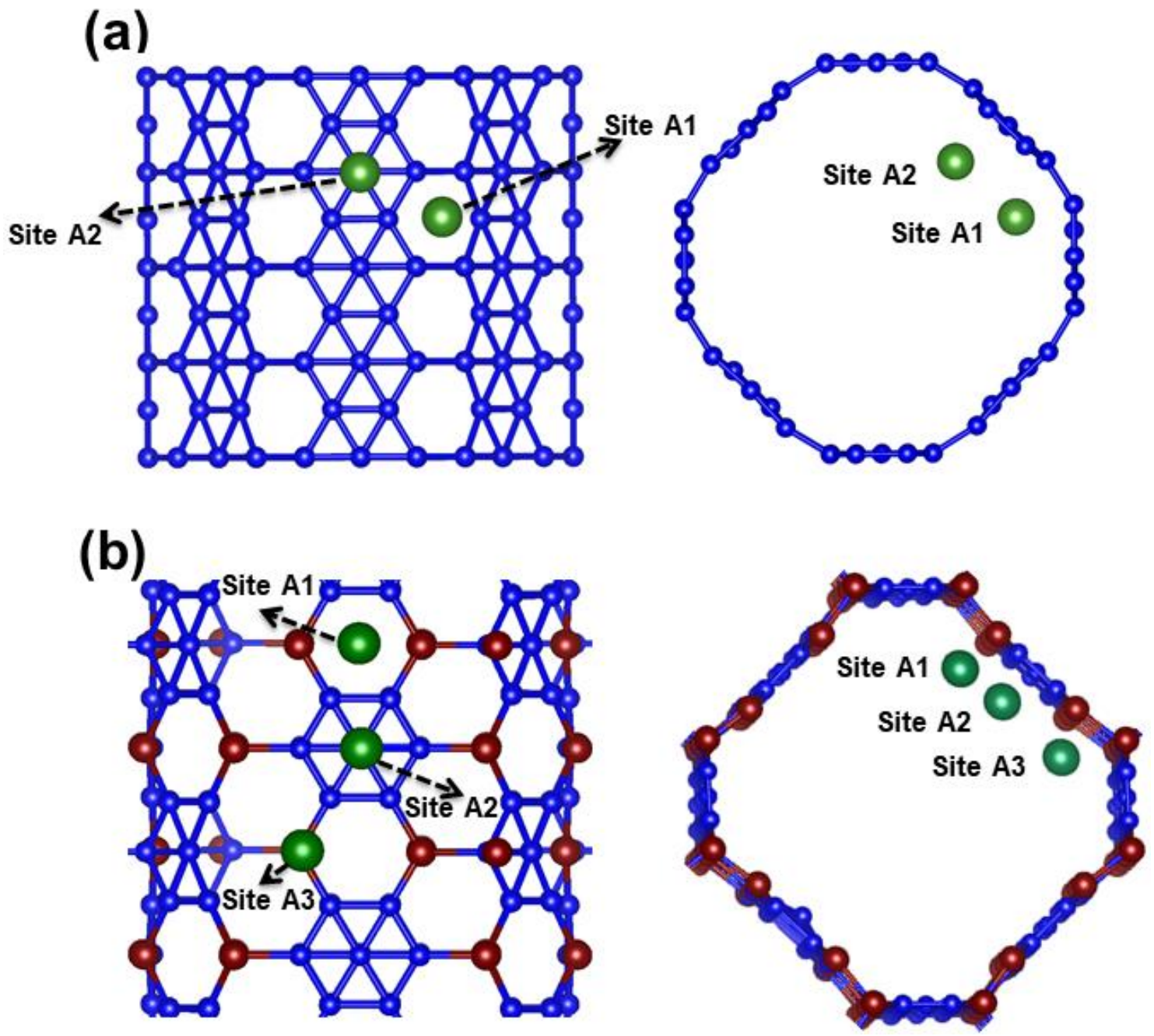

Figure S3. Active sites for $\mathrm{Li}, \mathrm{Na}$, and $\mathrm{K}$-ions in (a) $3 \mathrm{D}-\beta_{12}$-borophene and (b) $3 \mathrm{D}-\mathrm{B}_{7} \mathrm{P}_{2}$. 
Table S2. Electron Density ( $\rho$ in atomic unit (atomic units)) and Laplacian of the Electron Density $\left(\nabla^{2} \rho\right.$ in atomic units) for Different BCPs after Ion Adsorption on 3D- $\beta_{12}$-borophene and 3D-B $\mathrm{P}_{2}$.

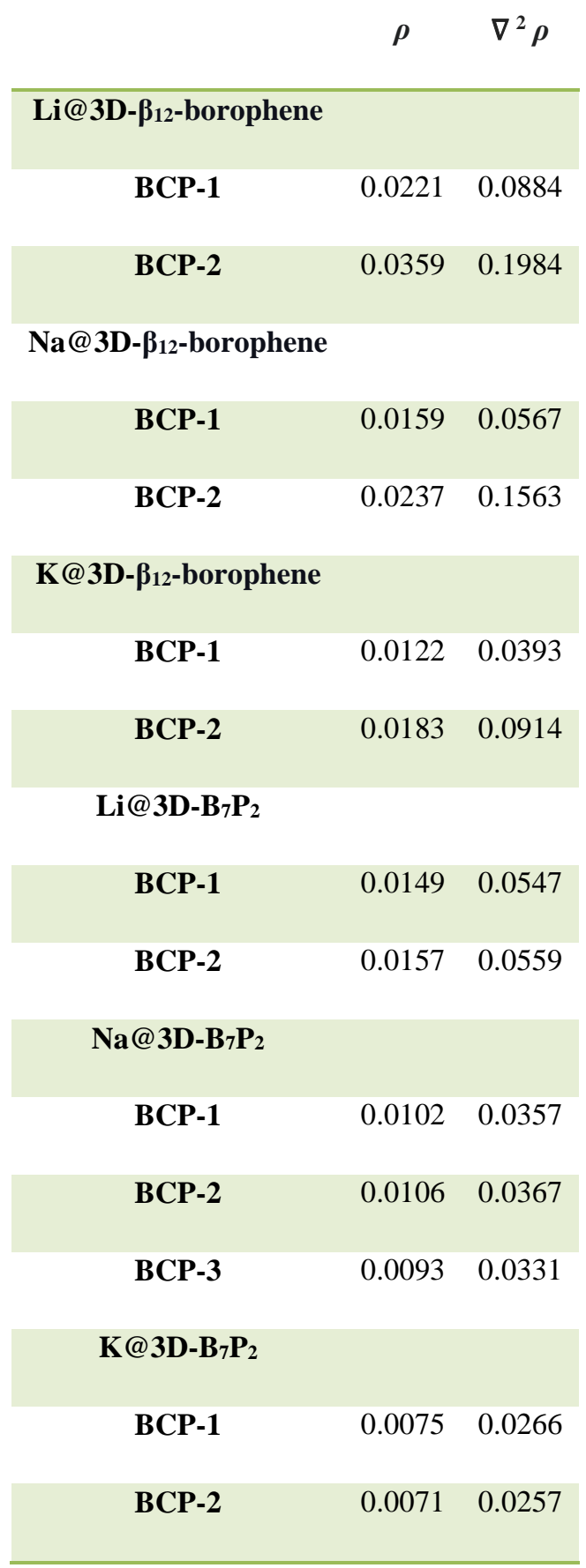




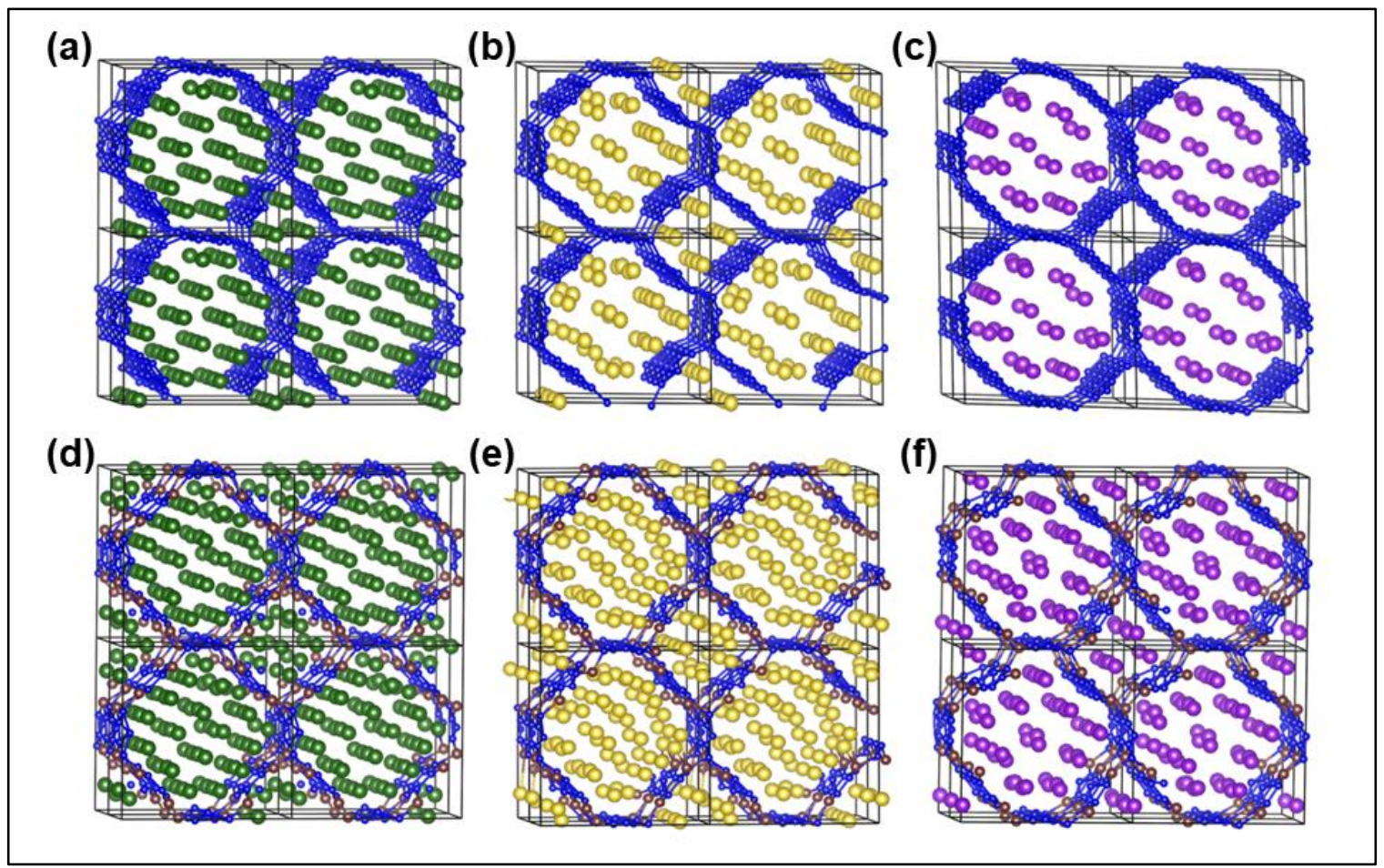

Figure S4. Geometric structures of (a-c) $3 \mathrm{D}-\beta_{12}$-borophene and (d-f) $3 \mathrm{D}-B_{7} P_{2}$ with the maximal number of metal ions adsorbed after $10 \mathrm{ps}$ AIMD simulations at $300 \mathrm{~K}$, where green, yellow, and purple spheres represent $\mathrm{Li}, \mathrm{Na}$, and $\mathrm{K}$, respectively.

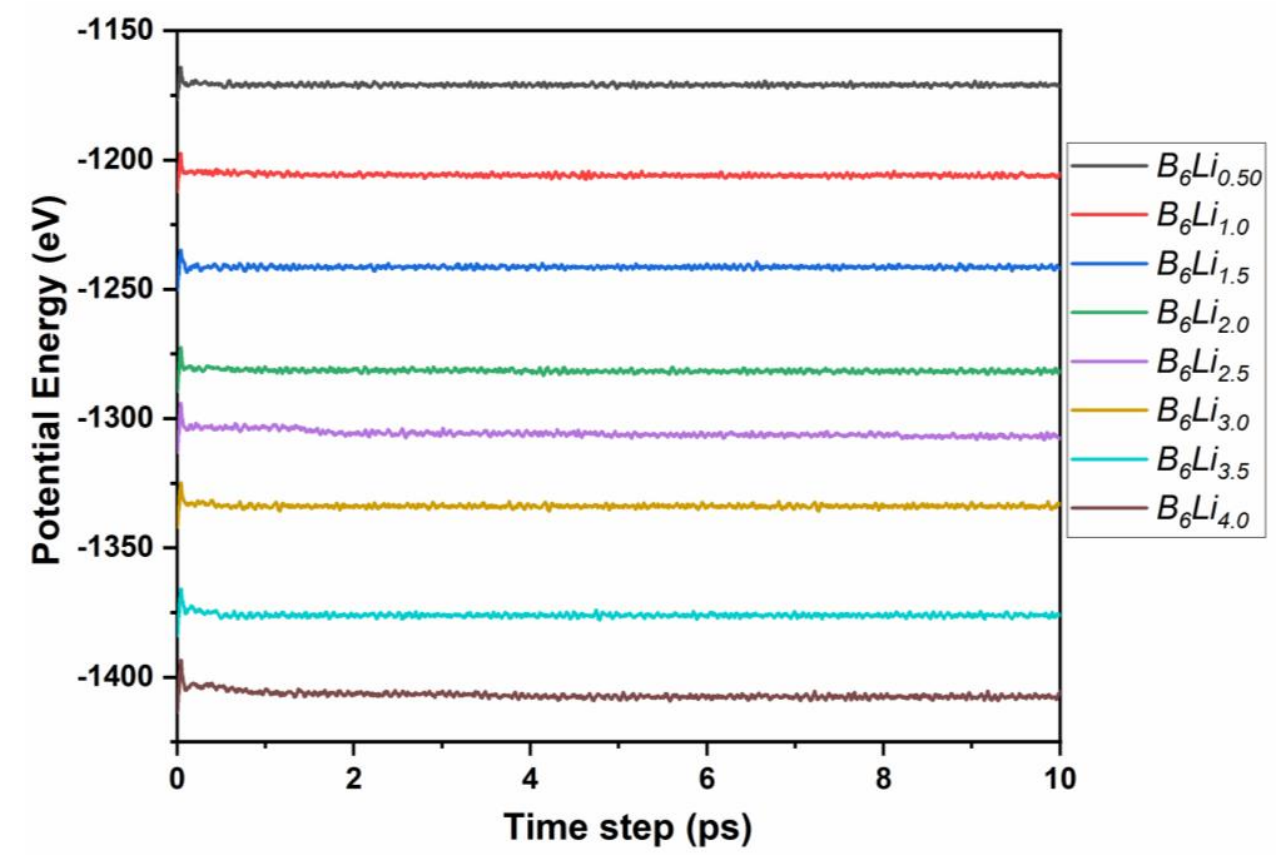

Figure S5. Fluctuations of the potential energy for the stable intermediate Li-phases of 3D- $\beta_{12}$-borophene during AIMD simulations at $300 \mathrm{~K}$. 


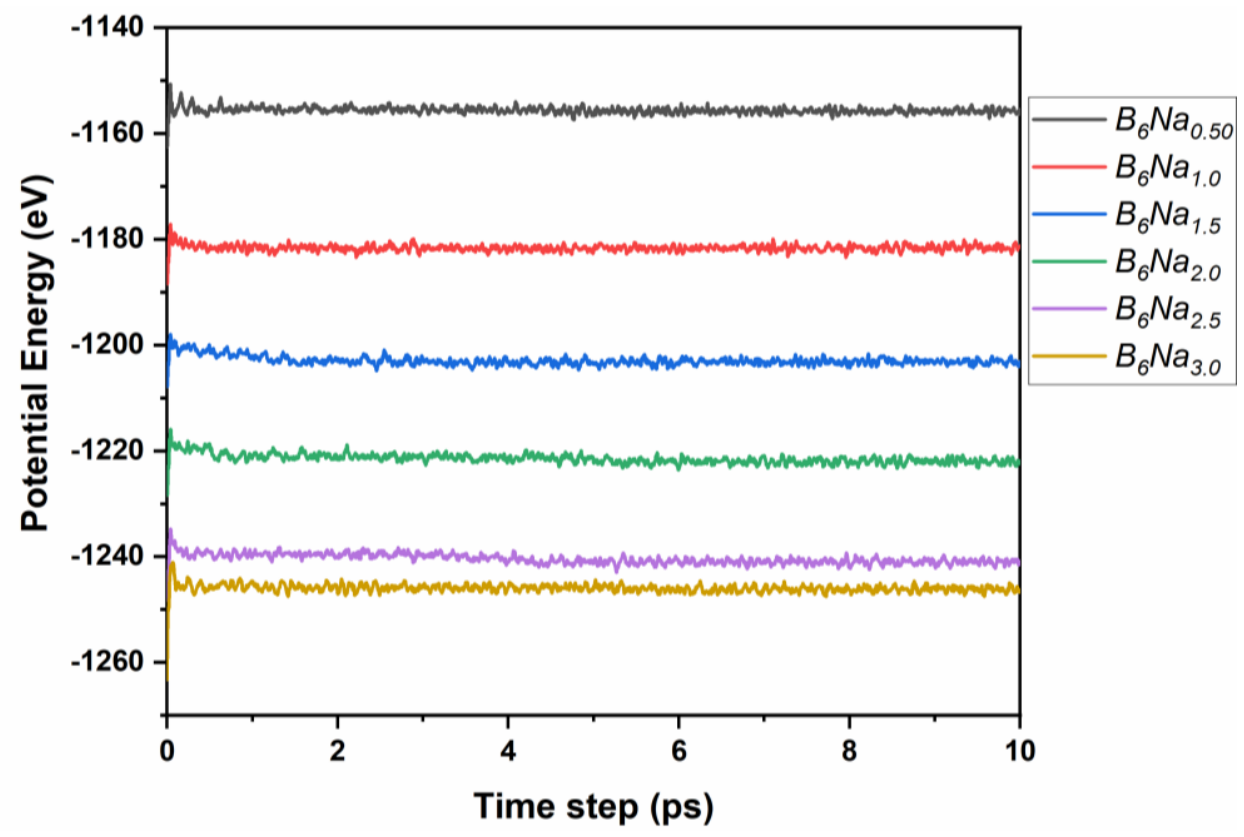

Figure S6. Fluctuations of the potential energy for the stable intermediate Na-phases of 3D- $\beta_{12}$-borophene during AIMD simulations at $300 \mathrm{~K}$.

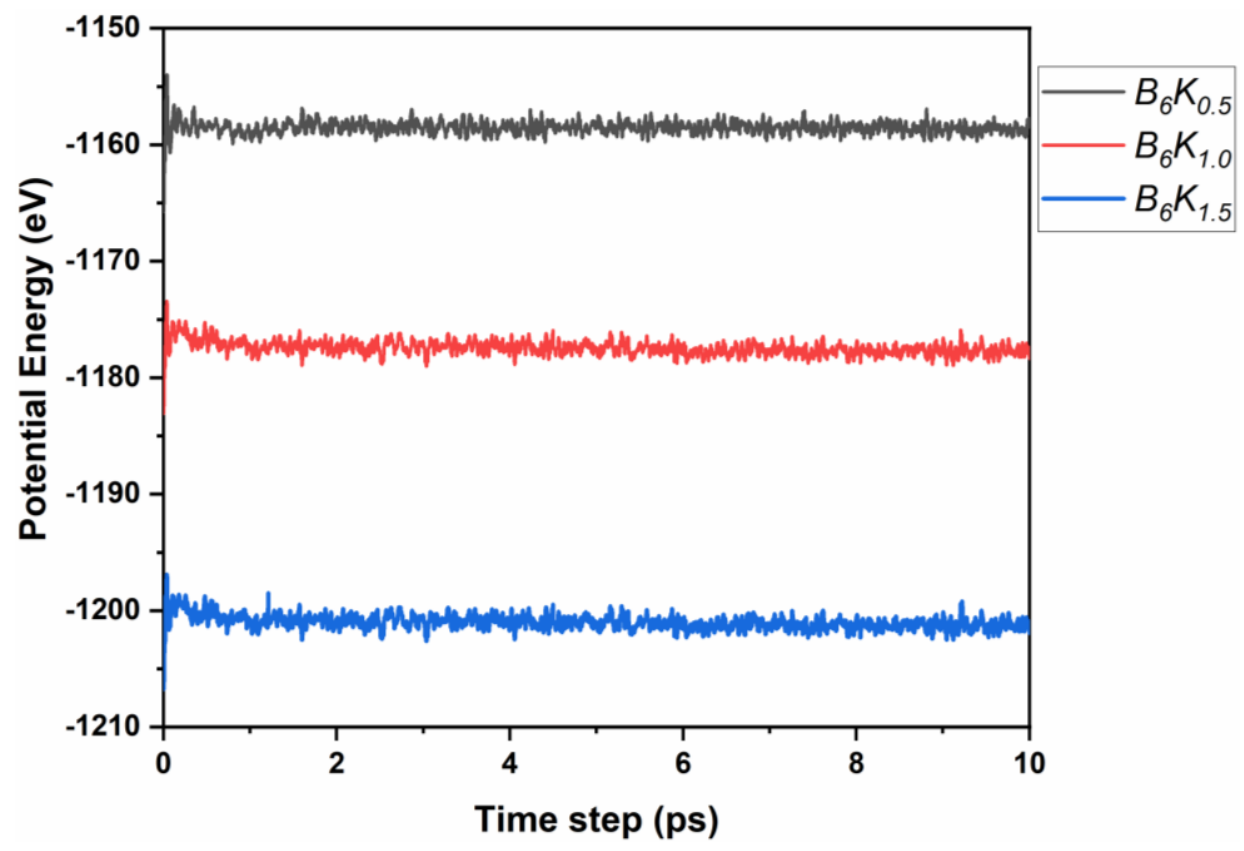

Figure S7. Fluctuations of the potential energy for the stable intermediate K-phases of 3D- $\beta_{12}$-borophene during AIMD simulations at $300 \mathrm{~K}$. 


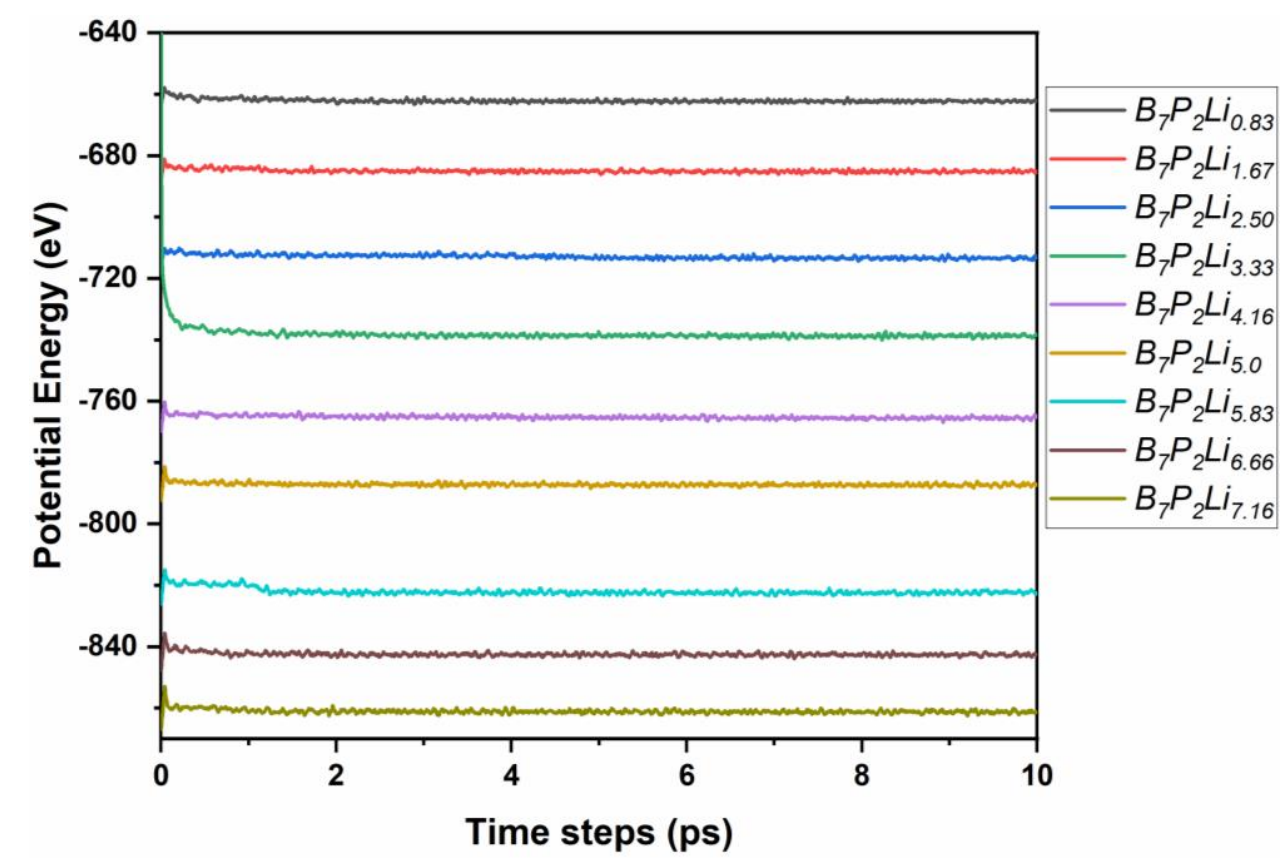

Figure S8. Fluctuations of the potential energy for the stable intermediate Li-phases of $3 \mathrm{D}_{-} \mathrm{B}_{7} \mathrm{P}_{2}$ during $\mathrm{AIMD}$ simulations at $300 \mathrm{~K}$.

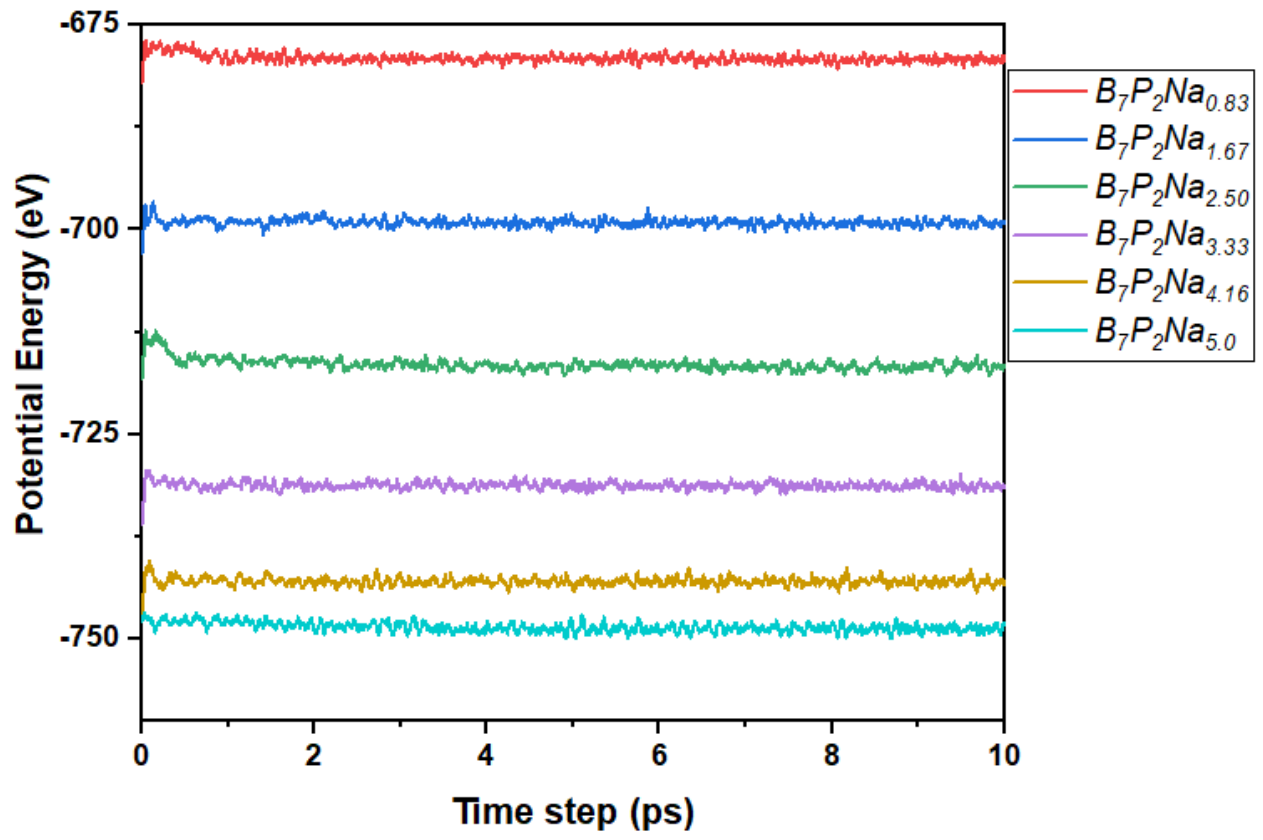

Figure S9. Fluctuations of the potential energy for the stable intermediate Na-phases of $3 \mathrm{D}_{-} \mathrm{B}_{7} \mathrm{P}_{2}$ during $\mathrm{AIMD}$ simulations at $300 \mathrm{~K}$. 


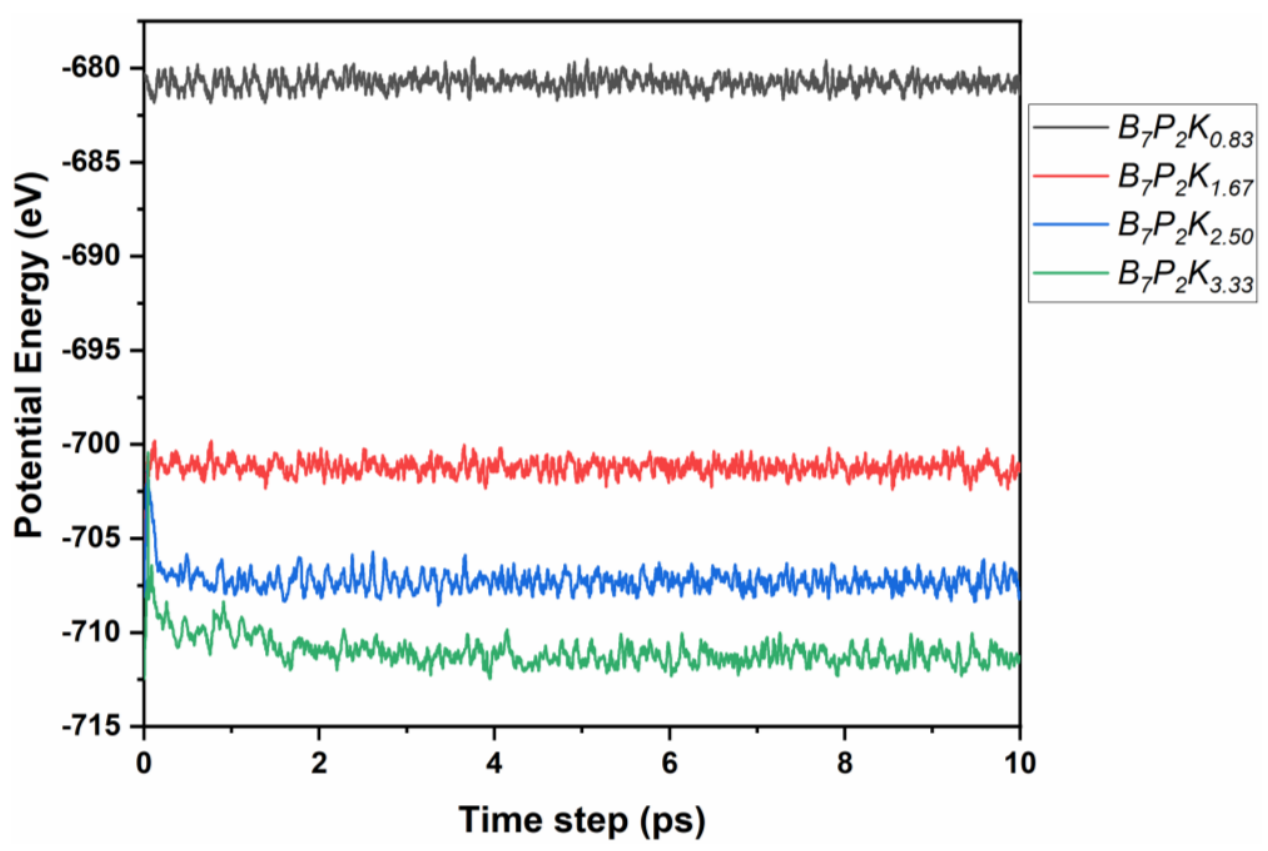

Figure S10. Fluctuations of the potential energy for the stable intermediate K-phases of $3 \mathrm{D}-\mathrm{B}_{7} \mathrm{P}_{2}$ during AIMD simulations at $300 \mathrm{~K}$.

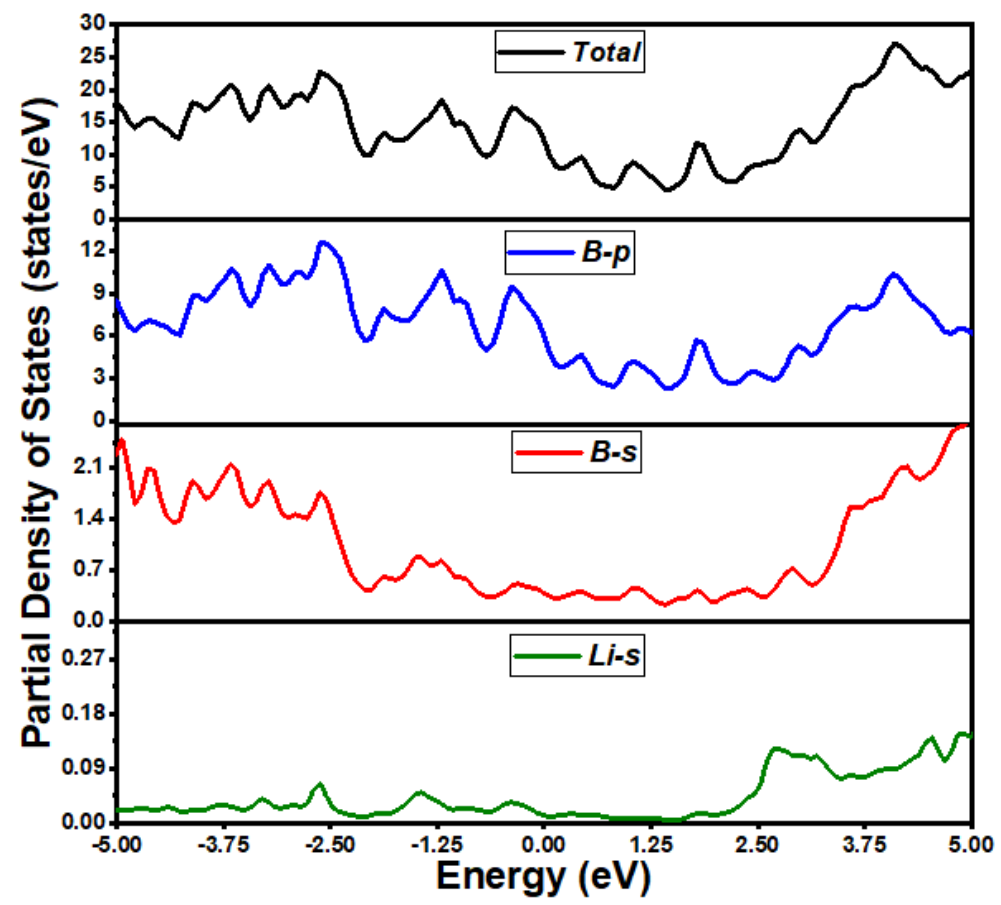

Figure S11. PDOS for single Li-ion adsorbed 3D- $\beta_{12}$-borophene. 


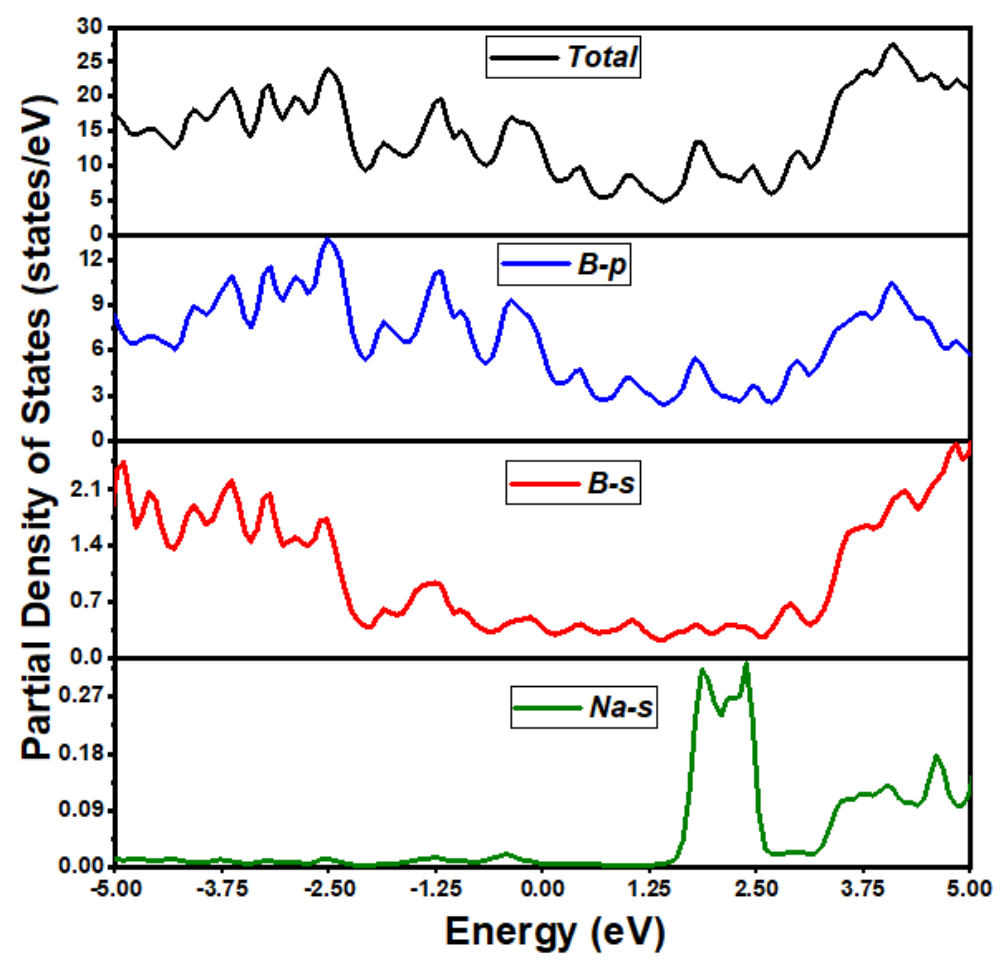

Figure S12. PDOS for single Na-ion adsorbed 3D- $\beta_{12}$-borophene.

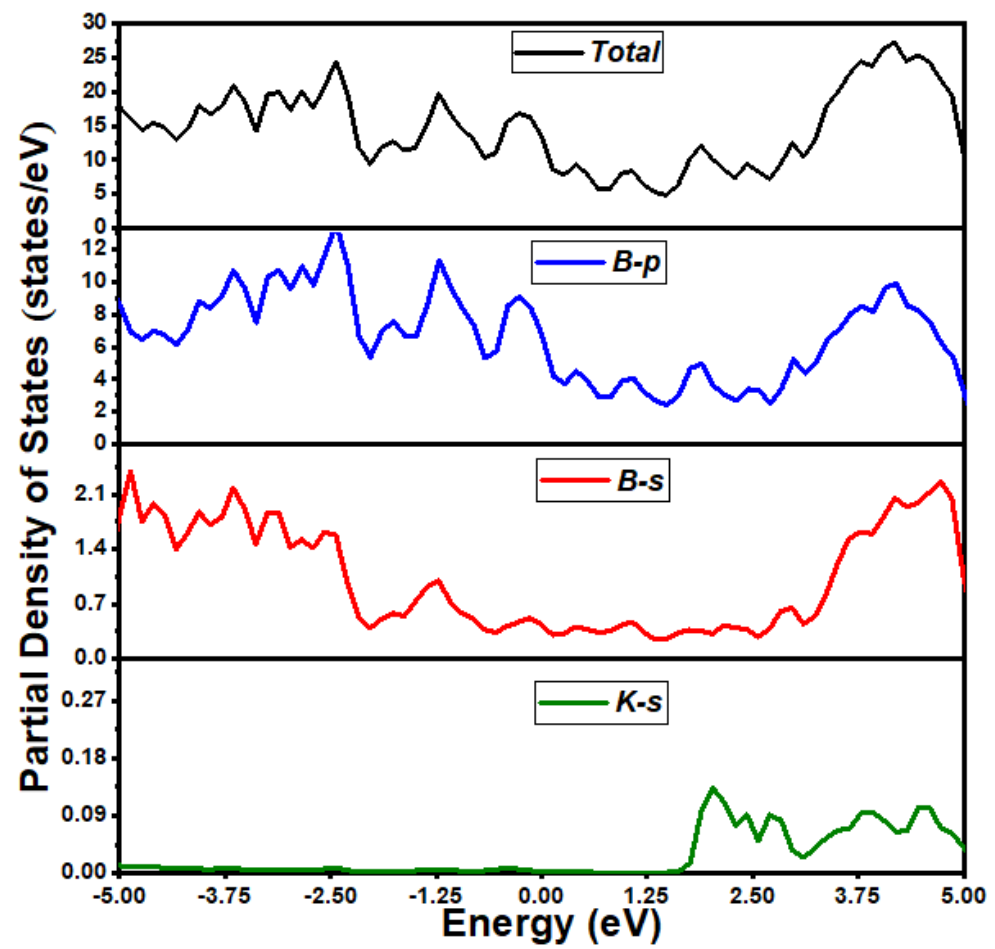

Figure S13. PDOS for single K-ion adsorbed 3D- $\beta_{12}$-borophene.. 


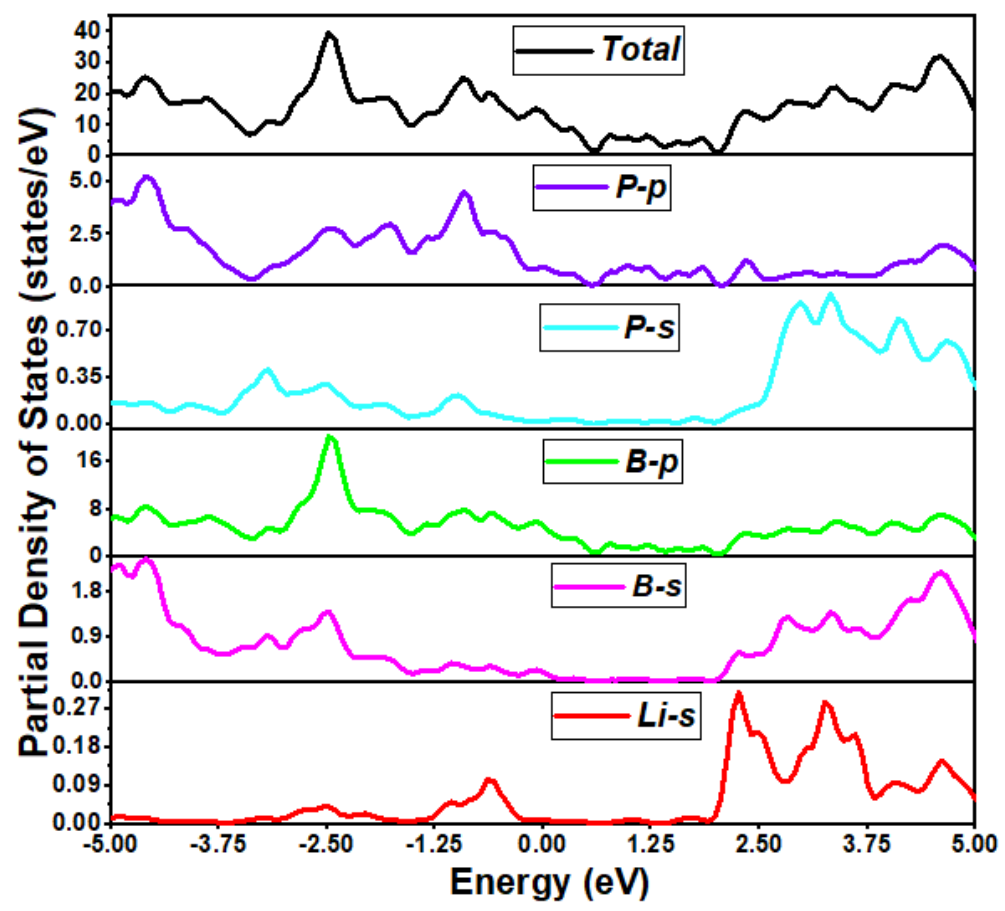

Figure S14. PDOS for single Li-ion adsorbed $3 \mathrm{D}-\mathrm{B}_{7} \mathrm{P}_{2}$.

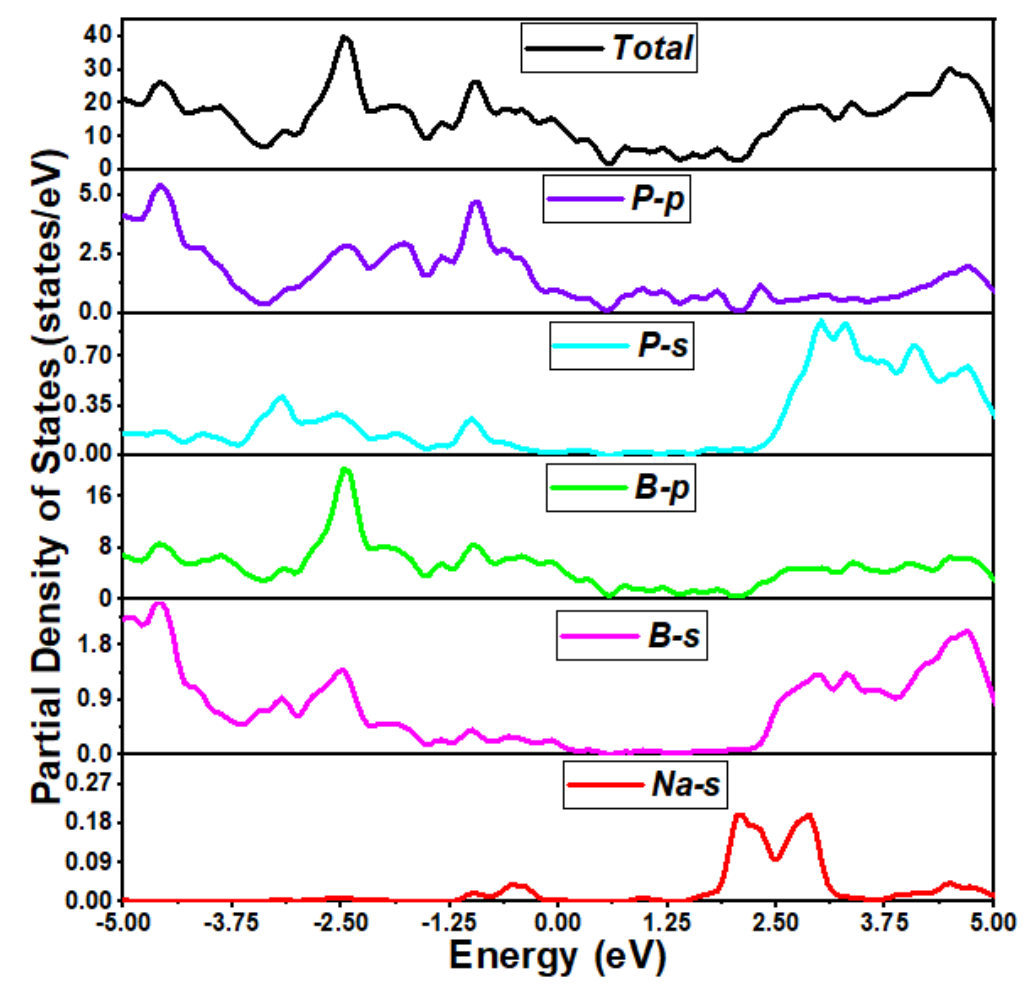

Figure S15. PDOS for single Na-ion adsorbed $3 \mathrm{D}-\mathrm{B}_{7} \mathrm{P}_{2}$. 


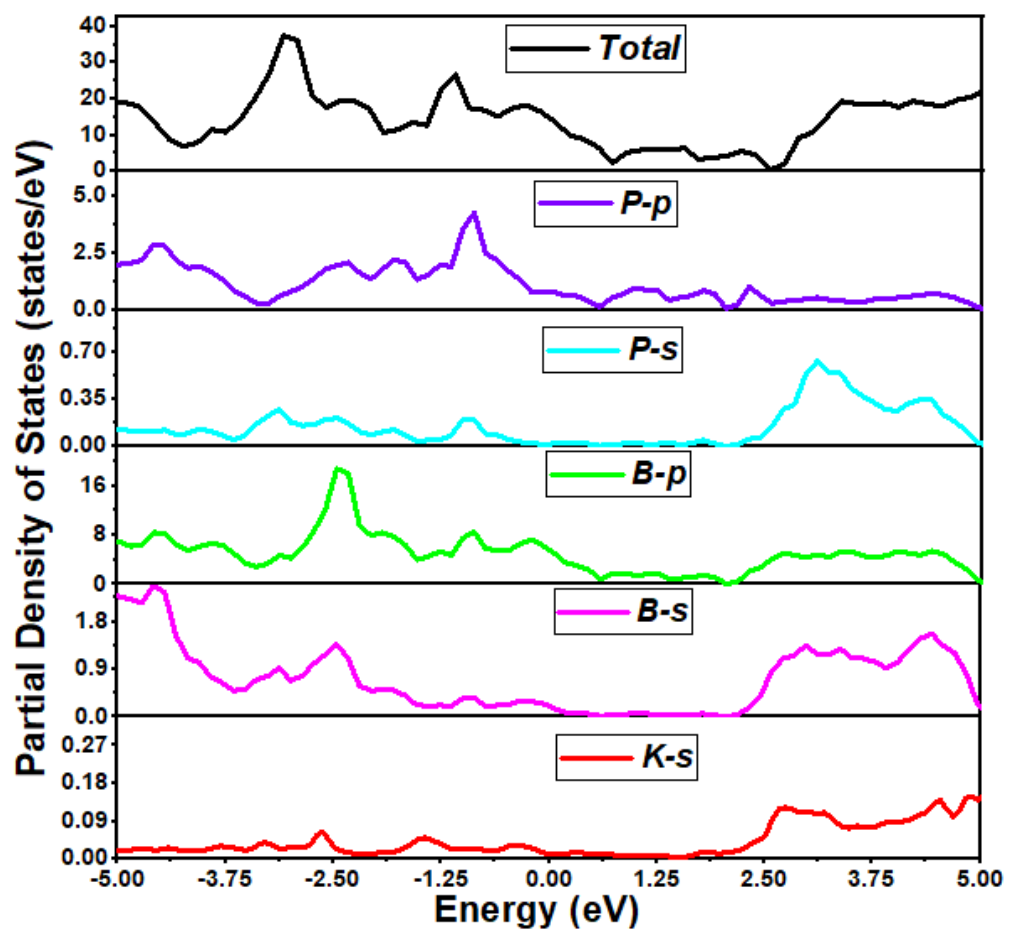

Figure S16. PDOS for single K-ion adsorbed $3 \mathrm{D}-\mathrm{B}_{7} \mathrm{P}_{2}$. 